\title{
La simulazione degli scambi acque superficiali/acque sotterranee: alcuni codici numerici a confronto
}

\author{
Iacopo Borsi \\ Dipartimento di Matematica - Università degli Studi di Firenze \\ borsi@math.unifi.it
}

\author{
Rudy Rossetto \\ Istituto di Scienze della Vita - Scuola Superiore Sant'Anna \\ r.rossetto@sssup.it
}

Negli ultimi anni il panorama della simulazione numerica del ciclo idrogeologico è andato sempre più arricchendosi di codici per analizzare gli scambi tra acque sotterranee e acque superficiali. Questo per soddisfare l'esigenza di strumenti per dare risposte agli impatti prodotti dai cambiamenti climatici in atto e, conseguentemente, alla necessità di pianificazione e gestione della risorsa idrica. Nell'analizzare i diversi approcci per la risoluzione delle acque superficiali, la principale differenza che si incontra è quella relativa all'utilizzo o meno dell'equazione di Saint-Venant (SV) per il calcolo dei flussi, siano essi relativi al ruscellamento o a corsi d'acqua. Il sistema di equazioni di SV si basa sulla conservazione della massa e del momento per descrivere il deflusso in un canale (Furman 2008). Di seguito è riportata una sintesi dei due approcci più comuni.

Bilanci di massa (BM): alcuni codici si limitano a calcolare bilanci di massa per l'acqua, riferiti a ciascuna cella di discretizzazione e a un particolare intervallo di tempo: la sommatoria del volume di acqua in eccesso o difetto, viene utilizzata per il calcolo del flusso (uscente o entrante) per la cella e diretto verso le celle adiacenti. Nel calcolo del bilancio sono considerati processi quali l'evapotraspirazione e l'intercettazione dovuta alla vegetazione, spesso con opzioni nella scelta delle formule da utilizzare per il loro calcolo. Di questa tipologia fanno parte, ad esempio, i codici SWAT (Neitsch et al. 2005) e PRMS (Leavesley et al., 1983), sviluppato prima autonomamente e successivamente integrato con alcune modifiche nell'ambiente GSFLOW (Markstrom et al. 2008).

Equazione di Saint-Venant (SV): i codici che invece utilizzano il sistema di equazioni di SV si differenziano principalmente nel trattamento del sistema stesso, nella sua versione completa (SVC), oppure introducendo alcune semplificazioni. Fra esse, la più diffusa è quella denominata kinematic wave (SVKW), che sostanzialmente implica il trascurare i termini di diffusione e di inerzia (come in ParFlow, Kollet e Maxwell., 2006). Altra approssimazione consiste nel trascurare solo i termini di inerzia, es. CATHY (Camporese et al. 2010). Tutti i codici esaminati utilizzano il sistema 2D (SV2D), ma lo limitano alla formulazione 1D nel caso di simulazione di corsi d'acqua, poiché in tal caso la direzione principale di flusso è una sola. Di questa categoria fa parte il codice PIHM (Yizhong, 2004). Altri codici invece risolvono il sistema in una sola dimensione spaziale (SV1D).

Per quel che riguarda il modo di descrivere la ricarica delle acque sotterranee, la classificazione è assai più semplice. Infatti, possiamo individuare sostanzialmente quattro diversi metodi:

1. l'utilizzo di semplici relazioni algebriche per il calcolo della velocità di infiltrazione. Indicheremo con RA la sigla relativa a questo tipo di approccio, che ad esempio è implementato in SWAT (Neitsch et al. 2005);

2. la risoluzione della sola equazione nella zona satura. In tal caso il flusso di ricarica proveniente dalla zona insatura viene introdotto come termine di sorgente nell'equazione di falda. E' questo il metodo utilizzato ad esempio dal pacchetto RECHARGE nel codice MODFLOW (McDonald and Harbaugh 1988);

3. la risoluzione dell'equazione di Richards 1D (R1D), che poi viene accoppiata alla risoluzione dell'equazione di governo delle acque sotterranee. In tal caso il reale problema 3D per l'equazione di Richards è suddiviso in due sotto-domini (la zona insatura e quella satura) nel primo dei quali il moto viene supposto puramente verticale;

4. la risoluzione completa dell'equazione di Richards definita sull'unico dominio. In tal caso l'individuazione della tavola d'acqua viene ottenuta non a priori, ma a posteriori come linea di livello della soluzione corrispondente al carico idraulico nullo. La sigla R3D contraddistinguerà questo caso.

Tre categorie possono essere definite circa i diversi approcci per la risoluzione dell'accoppiamento:

- disaccoppiamento (DA): i due sistemi (superficiali e sotterraneo) vengono risolti separatamente ad ogni passo temporale. Tipicamente prima viene risolto il sistema superficiale (poiché la sua dinamica è più veloce di quella del sotterraneo) imponendo che all'interfaccia avvenga un flusso di tipo Darcy, funzione della differenza tra il carico idraulico calcolato superficiale e sotterraneo, moltiplicata per la conduttanza. In questa formula dunque è presente anche la soluzione dell'equazione per le acque sotterranee, che però non è nota a quel passo temporale. Per ovviare a ciò, generalmente al posto di essa viene presa quella del passo temporale precedente;

- accoppiamento iterativo (AI): nel metodo precedente, una volta risolta l'equazione per le acque superficiali, essa viene passata all'altro sistema come condizione al contorno per risolvere in modo completo quel passo temporale. Una maggiore accuratezza può essere raggiunta aggiungendo un loop di iterazione a questo livello per verificare se le soluzioni così calcolate siano coerenti con la condizione assegnata. In altre parole, in questo metodo viene eseguito il calcolo del metodo precedente sin quando 
la differenza fra le soluzioni a due iterazioni successive non risulti inferiore a una tolleranza. Quindi, sebbene le equazioni siano sempre disaccoppiate, l'errore che si commette nel disaccoppiamento viene controllato e diminuito dal processo iterativo.

- accoppiamento completo (AC): il sistema viene risolto nella sua interezza, ad ogni passo temporale. E' evidente che questo approccio, pur essendo di maggior precisione numerica, richiede costi computazionali notevolmente maggiori. Si veda ad esempio Bittelli et al. (2010)

La Tabella 1 sintetizza questa classificazione e può essere utile a chi voglia avvicinarsi a questo tipo di strumenti. Tuttavia, occorre precisare che, oltre alla conoscenza dei dettagli tecnici è indispensabile avere ben chiari alcuni aspetti di tipo "pratico", quali la scelta di rivolgersi a codici free o licenze commerciali, possibilità di disporre di GUI per il pre- e postprocessing, tempi di calcolo necessari alla simulazione del problema in analisi.

Tab.1 - Tabella di comparazione dei codici presi in esame. Note. (a): MODFLOW - 2005 accoppiato con PRMS. (b): Integrato in MODFLOW - 2000.

\begin{tabular}{|c|c|c|c|c|}
\hline Nome & Acque sup. & Zona Insatura & Accoppiamento & $\begin{array}{l}\text { Riferimento } \\
\text { bibliografico }\end{array}$ \\
\hline GSFLOW $^{(\mathrm{a})}$ & $\mathrm{BM}+\mathrm{SV} 1 \mathrm{D}$ & R1D, FDM & AI & Markstrom et al., 2008 \\
\hline SWAT & $\mathrm{BM}$ & -- & -- & Neitsch et al., 2005 \\
\hline SUTRA & -- & R3D, FEM & -- & Voss and Provost, 2010 \\
\hline HydroGeoSphere & SV2D,FEM & R3D,FEM & AC & Brunner et al., 2012 \\
\hline $\mathrm{VZF}^{(\mathrm{b})}$ & -- & R3D, FDM & -- & Thoms et al., 2006 \\
\hline PHIM & SV2D, FVM & R3D, FVM & AC & Yizhong Qu , 2004 \\
\hline MIKE-SHE & SV2D, FDM & R1D,FDM & $\mathrm{AI}$ & Graham, 2005 \\
\hline ParFlow & SVKW, FVM & R3D, FVM & $\mathrm{AI}$ & Kollet e Maxwell., 2006 \\
\hline CATHY & SV1D, FDM & R3D, FEM & AI & Camporese et al., 2010 \\
\hline Criteria3D & SVC, & R3D, FVM & $\mathrm{AC}$ & Bittelli et al., 2010 \\
\hline
\end{tabular}

\section{BIBLIOGRAFIA}

Bittelli M., Tomei F., Pistocchi A., Flury M., Boll J., Brooks E.S. and Antolini G. (2010). Development and testing of a physically based, 3D model of surface and subsurface hydrology, Adv. Wat. Res., 33(1), 106-122.

Brunner, P., and C. T. Simmons (2012), HydroGeoSphere: A Fully Integrated, Physically Based Hydrological Model, Ground Water, 50(2), 170-176.

Camporese M., C. Paniconi, M. Putti, S. Orlandini, Surface-subsurface flow modeling with path-based runoff routing boundary condition-based coupling and assimilation of multisource observation data, Water Resour. Reser., 46 (2): 1-22, 2010

Clifford I. Voss and Alden M. Provost (2008), SUTRA, A Model for Saturated-Unsaturated, Variable-Density Ground-Water Flow with Solute or Energy Transport.

A. Furman. Modeling coupled surface-subsurface flow processes: A review. Vadose Zone J., 7(3):741--756, 2008

Graham, D.N. and M.B. Butts 2005. Flexible, integrated watershed modelling with MIKE-SHE. In Watershed Models, Eds. V.P. Singh $\&$ D.K. Frevert. CRC press.

S.J. Kollet and R.M. Maxwell (2006), Integrated surface-groundwater flow modeling: a free-surface overland flow boundary condition in a parallel groundwater flow model, Adv. Wat. Res. 29, 945-958.

Leavesley, G.H., Lichty, R.W., Troutman, B.M., and Saindon, L.G., 1983, Precipitation-runoff modeling system-User's manual: U.S. Geological Survey Water-Resources Investigations Report 834238, 207 p.
McDonald, M.G., and Harbaugh, A.W., 1988. A modular three-dimensional finite-difference ground-water flow model: U.S. Geological Survey Techniques of Water-Resources Investigations, book 6, chap. A1, 586 p.S.L. Neitsch, J.G. Arnold, J.R. Kiniry, and J.R. Williams (2005). Soil and Water Assessment Tool Theoretical Documentation vs 2005. http://swatmodel.tamu.edu/ [ultimo accesso 22/06/2012]

S.L. Markstrom, R.G. Niswonger, R.S. Regan, De.E. Prudic, P.M. Barlow (2008) GSFLOW - Coupled ground-water and surface-water \#ow model based on the integration of the Precipitation-Runo\# Modeling System (PRMS) and the Modular Ground-Water Flow Model (MODFLOW-2005): U.S. Geological Survey Techniques and Methods 6-D1.

R.B. Thoms and R.L. Johnson and R.W. Healy (2006), User's guide to the Variably Saturated Flow (VSF) Process for MODFLOW: U.S. Geological Survey Techniques and Methods 6-A18,

Voss, C. I., and Provost, A.M., (2010), SUTRA, A model for saturated-unsaturated variable-density ground-water flow with solute or energy transport, U.S. Geological Survey Water-Resources Investigations Report 02-4231.

Yizhong Qu (2004) An integrated hydrologic model for multi-process simulation using semi--discrete finite volume approach, The Pennsylvania State University, Thesis in Civil Engineering. 